\title{
Pautas para la medición de la estructura de la ciudad actual, en el marco del modelo urbano disperso, utilizando el catastro de urbana. Aplicación al municipio suburbano de Getafe, Madrid
}

\author{
José Miguel Santos Preciado
}

\section{Resumen}

La realidad de nuestras grandes urbes muestra cómo la ciudad compacta se está transformando en una ciudad cada vez más dispersa y fragmentada, con periferias más extensas y límites más difusos. La dispersión de la ciudad por el territorio ha estado acompañada, en general, de cierta pérdida de su tradicional complejidad, debido a la similitud funcional y separación espacial de las diversas partes que conforman su estructura urbana.

En este contexto, el uso del catastro de urbana, como repositorio de datos espaciales, resulta de gran interés en la investigación funcional de la ciudad. El trabajo que presentamos tiene como objetivo definir una metodología aproximativa a la identificación tipológica de unidades estructurales básicas de la ciudad actual, en el municipio suburbano de Getafe (Madrid), a partir de la combinación potencial de los usos del suelo existentes en cada parcela catastral.

Palabras clave: Cartografía catastral. Sistemas de Información Geográfica. Estructura urbana. Desarrollo urbano.

\section{Abstract}

The reality of our big cities shows how the compact city is being transformed into a city always more disperse and fragmented, with more extensive outskirts, and more diffuse limits. The scattering of the city through the territory has been accompanied, in general, with a certain loss of its traditional complexity, due to the functional similarity and the spatial separation of the different parts which comprise its urban structure.

In this context, the use of the urban land registry as a repository of spatial data, is 
of great interest in the functional investigation of the city. The work that we present aims to define a methodology that approximates to the typological identification of basic structural units of the modern city, in the suburban municipality of Getafe (Madrid), starting from the potential combination of existing uses of each plot of land in the land registry.

Keywords: Cartography of the registry. Geographical Information Systems. Urban structure. Urban development.

José Miguel Santos Preciado (jsantos@geo.uned.es), Departamento de Geografía, Universidad Nacional de Educación a Distancia (UNED), Madrid (España). 


\section{INTRODUCCIÓN}

Desde hace algunos años, estamos asistiendo a un nuevo fenómeno que tiende a transformar la realidad de las ciudades del mundo desarrollado. "A escala municipal, metropolitana o regional es evidente que existe una dispersión de la urbanización sin precedentes, y que las dinámicas urbanas integran, funcionalmente, espacios construidos que no tienen continuidad física entre ellos y, a menudo, se encuentran, incluso, a muchos kilómetros de distancia" (Nel.lo, 1998), lo que ha llevado a referirse al mismo con el término de "ciudad dispersa".

Sin embargo, algunas de estas transformaciones se están realizando en la ciudad europea según patrones particulares, que tienen más que ver con sus propias coordenadas históricas y culturales. En el caso de España, entre las características más visibles de los nuevos tiempos habría que destacar que la configuración de la periferia de nuestras ciudades, tradicionalmente afín al modelo compacto de ciudad, se estaría produciendo, en buena parte, con la proliferación de un tejido residencial extensivo de viviendas unifamiliares, aunque no de forma generalizada, apreciándose estándares de edificabilidad y densidad de ocupación muy contrastados, según los sectores de la ciudad. A ello habría que añadir, la localización periférica del comercio y las oficinas, antes centralizados. Este proceso descentralizador de la actividad urbana no habría sido posible sin el desarrollo de un sistema de transportes reticular, que permite la interrelación de zonas cada vez más separadas entre sí, así como por la utilización masiva del automóvil privado por la población residente en la ciudad.

Este proceso de cambio se ha producido desde una profunda transformación funcional de los elementos que componen la estructura de las grandes regiones urbanas. La organización territorial de la ciudad tradicional, caracterizada por la complejidad morfo tipológica y la mezcla de usos del suelo, está dando paso a un nuevo territorio urbano donde "las formas espaciales emergentes, en que se materializan los nuevos nodos de acumulación, comparten una serie de rasgos comunes, donde destaca el carácter especializado y frecuentemente mono funcional o monotemático" de los fragmentos que componen el nuevo mosaico de la ciudad actual (De Santiago, 2008).

Esta realidad dual se percibe en el profundo cambio sufrido por las áreas periféricas de las grandes ciudades españolas. En este sentido, pretendemos mostrar alguna de las transformaciones funcionales experimentadas en Madrid, una de nuestras urbes más emblemáticas, para lo que hemos seleccionado Getafe, municipio suburbano de la primera corona metropolitana. En este sentido, nos proponemos, como apoyo a la aplicación de la metodología que vamos a exponer, el aná- 
lisis comparativo de algunas áreas surgidas en el citado municipio, en momentos diferenciados en el tiempo. Para ello, utilizaremos la información georreferenciada de los datos del catastro de urbana, relativa a las coberturas de distintas variables de ocupación del suelo.

Esta labor la hemos llevado a cabo, metodológicamente, en dos etapas. En la primera, se ha tratado de definir una tipología de unidades estructurales básicas, a partir de la combinación potencial de los usos del suelo existentes en cada parcela catastral. El objetivo fundamental se ha concretado en diseñar un procedimiento taxonómico, dirigido a manipular y organizar un variado conjunto de variables, relacionadas con los usos del suelo, capaces de generar una cartografía que ofrezca respuesta a la problemática de la localización de actividades u ocupaciones en el territorio. La segunda etapa ha estado dirigida a la reconstrucción del patrón de unidades estructurales derivadas, a partir de las anteriores, mediante la integración contigua de unidades territoriales de similar contenido temático, de acuerdo a los patrones definidos anteriormente.

\section{LAS BASES DE DATOS CATASTRALES Y SU APLICACIÓN AL ESTUDIO DE LA ESTRUCTURA URBANADE GETAFE (MADRID)}

El éxito o fracaso de una investigación está relacionado, en buena parte, con la disponibilidad o no de una base de datos precisa, fiable y de calidad, lo suficientemente flexible como para alcanzar los objetivos propuestos en una escala espacial operativa. Así, hace relativamente poco tiempo, se ha puesto a disposición pública un volumen considerable de información georreferenciada, de especial interés para llevar a cabo diferentes tareas relacionadas con la aplicación de las Tecnologías de la Información Geográfica (TIG) en el análisis y evaluación de diferentes aspectos relacionados con el actual modelo territorial. En este sentido, el catastro de urbana, elaborado por la Dirección General del Catastro del Ministerio de Hacienda y Administraciones Públicas de España, dispone de una información de suficiente nivel de detalle y cualificación ${ }^{1}$ como para alcanzar un objetivo como el que nos proponemos. Dicho repositorio y sus unidades espaciales de referencia (principalmente la parcela catastral) ha resultado fundamental en nuestra investigación, ya que contiene información muy variada de las unidades fiscales que integran (usos del suelo, complementado por las variables de superficie ocupada, superficie edificada, momento de la construcción, etc.).

La utilización de la parcela catastral en los estudios urbanos encuentra su justifi-

\footnotetext{
${ }^{1}$ En nuestra investigación, hemos empleado la base de datos del catastro de urbana de la Comunidad de Madrid, disponible en 2009, cedido por la Dirección General del Catastro del Ministerio de Hacienda.
} 
cación en constituir un ente geográfico homogéneo, cuya naturaleza intrínseca se deriva de su particular relación con la propiedad, así como de su contenido, relativo a sus características morfológicas, temporales y funcionales. Los datos temáticos que contienen dichas unidades espaciales se refieren, tanto a variables de tipo físico, que atienden a las dimensiones de las parcelas, superficie del solar y tipo de parcela, como a variables morfológicas, relacionadas con las características particulares de la trama urbana, variables temporales, que reflejan los momentos de comienzo y finalización en la construcción de los edificios que integran cada parcela, y, finalmente, variables de tipo funcional, que responden al uso y destino de la edificación, de forma diferenciada para cada tipo de construcción².

Figura 1. Parcelas catastrales del municipio de Getafe (Madrid).

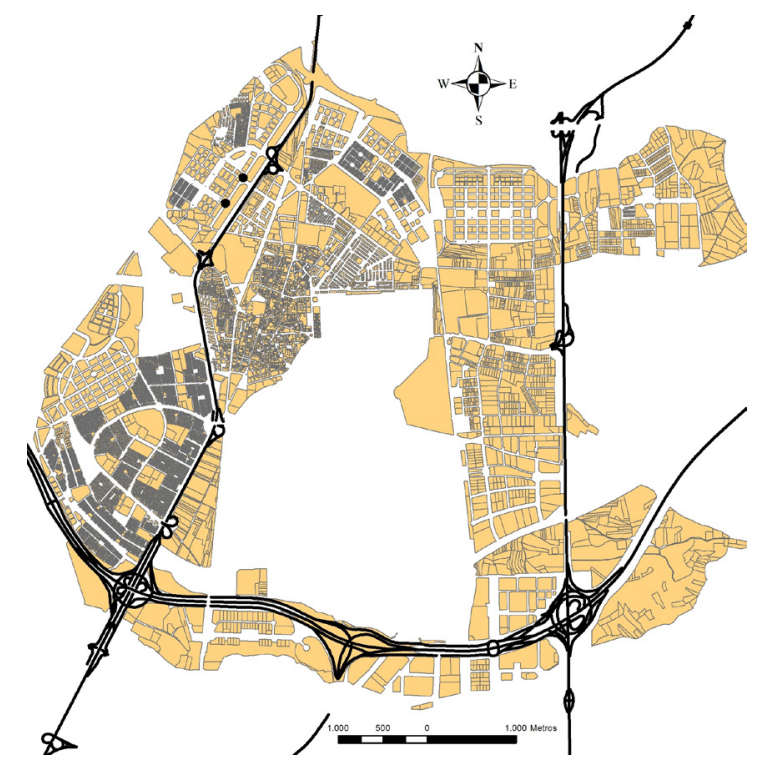

En nuestro caso, la parcela catastral se ha convertido en un elemento importante para el estudio e interpretación de la ciudad, al permitir relacionar su crecimiento con la estructura y morfología urbanas connaturales con el mismo. Con el fin de alcanzar el objetivo propuesto, hemos utilizado la cartografía catastral urbana del municipio de Getafe, perteneciente al sector suroeste de la Comunidad de Madrid. La representación cartográfica del mismo puede apreciarse en la Figura 1, enmar-

\footnotetext{
2 Las categorías identificadas son las siguientes: almacén-estacionamiento; residencial; industrial; oficinas; comercial; deportivo; espectáculos; ocio y hostelería; sanidad y beneficencia; cultural; religioso; obras de urbanización y jardinería; suelos sin edificar; edificio singular; almacén agrario; industrial agrario; agrario.
} 
cada su situación respecto a las principales vías de comunicación por carretera de la región.

\section{DEFINICIÓN DE UNIDADES FUNCIONALES BÁSICAS EN LAESTRUCTURA DE LA CIUDAD DE GETAFE (MADRID)}

Las transformaciones funcionales, resultado de la modificación acaecida en la organización espacial de la producción y el consumo, han sido determinantes para concebir un cambio intenso y definitivo en la estructura actual de la ciudad. Entre los aspectos centrales a considerar al estudiar la reorganización de los usos del suelo, cabe mencionar diversos enfoques, según los fines y propósitos de cada investigación particular. A partir de Rhind y Hudson (1980), se han planteado, al respecto, tres perspectivas metodológicas diferentes: a) un enfoque funcional, según el cual, el uso del suelo se define en función de la actividad que se desarrolle en él; b) un enfoque formal, que identifica el uso del suelo con determinadas características derivadas del aspecto visual de su representación: el tono, la textura, la densidad, la forma, el color, etc.; y c) un enfoque multidimensional, que supone la combinación de la funcionalidad de los usos y de las características visuales de los mismos.

En el análisis que planteamos, nos hemos decantado por la primera de las tres opciones. Pero, ¿cómo plantear el estudio de la estructura de la ciudad, a partir del contenido funcional de unidades espaciales elementales? Hemos partido de la parcela catastral como unidad básica de referencia espacial. La parcela catastral tiene unas características diferentes en el aspecto funcional y/o en el aspecto físico, tanto desde el punto de vista cualitativo como desde el punto de vista cuantitativo. La particularidad de la misma se deriva de la posibilidad de constituir espacios multifuncionales; es decir, de integrar actividades y mezcla de usos del suelo complementarios.

Disponer de los datos geográficos (espaciales y temáticos), almacenados estructuralmente en el seno de una base de datos, aporta una gran flexibilidad en el uso que pueda hacerse de ellos. Este uso va más allá de la producción cartográfica e incluye determinadas labores de análisis, de gran interés en la toma de decisiones espaciales. En este sentido, dichas bases de datos, vinculadas a su manejo por los Sistemas de Información Geográfica (SIG), nos ha permitido la posibilidad de extraer información derivada por reelaboración de la ya existente. Esta nueva información ha estado relacionada con las combinaciones tipológicas dominantes de los usos del suelo, así como en la medición precisa del número de parcelas y suelo ocupado y edificado en cada una de las composiciones resultantes. 
De acuerdo con el objetivo del trabajo, la primera de las tareas ha consistido en la clasificación o taxonomía de los usos, mediante la desagregación y asociación de categorías resultantes de las parcelas en el territorio analizado. No todos los usos del suelo urbano tienen la misma representación en la ciudad. Atendiendo a su grado de implantación, hemos estimado como más representativos, por la especial vinculación que tienen con el desarrollo del planeamiento urbano, los siguientes:

- El uso del suelo residencial, correspondiente al alojamiento, con carácter permanente 0 no, de personas y sus familias. La gran superficie de suelo ocupado por esta función urbana, además de su variedad y papel desempeñado en la estructura de la ciudad, ha sido la causa de que apreciáramos nuevas categorías más pormenorizadas dentro del mismo, como la vivienda unifamiliar y la vivienda multifamiliar.

- El uso del suelo comercial, que comprende aquellas actividades destinadas a suministrar mercancías al público, o a prestar determinados servicios a los particulares.

- El uso del suelo industrial, que tiene por finalidad llevar a cabo las operaciones de elaboración, transformación, reparación, almacenaje, y distribución de productos manufacturados.

- El uso del suelo de oficinas, que corresponde a aquellas actividades terciarias que se dirigen, como función principal, a prestar servicios de carácter administrativo, técnico, financiero, de información u otra naturaleza, realizados a partir del manejo y transmisión de información y conocimientos, bien dirigidos a las empresas y/o los particulares.

En conjunto, resultan un total de cinco categorías a considerar (uso residencial de vivienda unifamiliar, uso residencial de vivienda multifamiliar, uso comercial, uso industrial y uso de oficinas), lo que supone una notable variedad en cuanto a la desagregación de categorías se refiere, con el propósito de realizar la clasificación de las parcelas catastrales por composición múltiple de los usos del suelo en ellas representados. El número de posibles agrupaciones de dichas categorías, de acuerdo a la teoría combinatoria, y a su existencia en la realidad en el caso que nos ocupa, ha resultado ser de 24 . Los resultados obtenidos mediante la realización de operaciones taxonómicas en un $\mathrm{SIG}^{3}$, relativos exclusivamente a las parcelas catastrales edificadas se pueden observar en la Tabla 1.

Las conclusiones más interesantes que pueden deducirse de un análisis de la tabla de distribución de las parcelas, de acuerdo a las diferentes categorías multifuncionales, son las siguientes:

3 Para ello se ha utilizado el SIG ArcGIS, en su versión 10.1 
1. El número de parcelas catastrales edificadas, de uso exclusivo (con un único uso del suelo), relativas a las cinco categorías de usos del suelo consideradas, suponen, para el municipio considerado, el $77,7 \%$ del total existente. La distribución parcial de los mismos muestra un desigual reparto, siendo el uso residencial unifamiliar el que prevalece sobremanera respecto a los demás (62,3\% del total). A gran distancia, le seguiría el uso industrial (7,5\%), el residencial multifamiliar $(6,9 \%)$, y, en menor medida, el comercial $(0,5 \%)$ y de oficinas $(0,5 \%)$.

2. Esta realidad queda matizada si atendemos a la superficie de suelo ocupada y al total de superficie edificada. En este sentido, el suelo industrial puro (muy importante en el municipio) alcanza el lugar preeminente de suelo ocupado superficialmente (396,2 ha), que podría verse incrementada si consideráramos las parcelas mixtas en las que aparece (12,1 ha adicionales). En segundo lugar, se hallaría el uso residencial unifamiliar (160,6 ha) y a mayor distancia el uso residencial multifamiliar (96,2 ha) y el comercial (52,5 ha). Respecto al suelo edificado, la función residencial alcanza las 676,4 ha de superficie, muy alejada del uso industrial (228,1 ha), el comercial (110,1 ha) y el de oficinas ( $51,5 \mathrm{ha})$.

3. El resultado de las posibles combinaciones de los usos del suelo muestra la existencia de parcelas con "usos del suelo mixto", que evidencian el nivel de complementariedad o rechazo de los mismos entre sí. Relativo al tejido residencial, podemos destacar que el uso destinado a la vivienda unifamiliar posee un carácter más puro, ya que apenas se asocia con el resto de usos (el 99,8\% del total de las parcelas son de uso exclusivo residencial). En este caso, las unidades territoriales destacan por su reducida extensión superficial relativa y su condición de escasa complementariedad respecto a otros usos del suelo. Como tendremos ocasión de mostrar, su comportamiento territorial resalta la tendencia al agrupamiento con otras unidades espaciales semejantes, de tamaño muy parecido, en unidades estructurales más amplias, de morfología edificatoria similar (aislada, pareada, adosada, etc.), fruto de operaciones urbanísticas unitarias.

4. No ocurre lo mismo con el suelo residencial multifamiliar, que se agrega con gran facilidad al uso comercial (el $43 \%$ de las parcelas comparten, en número, ambos usos del suelo, mientras que apenas un escaso 2,9\% lo hacen, de forma exclusiva, con el uso industrial y/o de oficinas). Esta asociación residencia/comercio es clásica, en el territorio analizado, de los cascos anti- 
guos y ensanches residenciales que tuvieron lugar en el primer momento de la conformación metropolitana de Madrid, en los municipios más próximos a la capital.

5. El comercio constituye el uso del suelo más promiscuo y de mayor capacidad de asociación con otros usos. Su relación natural con el tejido residencial multifamiliar forma parte de nuestra cultura, estando ligado, íntimamente, a la existencia de locales artesanales o mixtos en las plantas bajas de edificios multi vivienda. Sin embargo, frente al modelo comercial tradicional han surgido, en el marco del modelo de la ciudad dispersa, grandes centros comerciales de la periferia, desligados de la calle y localizados en la proximidad a las autopistas urbanas. La nueva estructura comercial agrupa no solo hipermercados, sino galerías comerciales anexas, donde se concentran boutiques y tiendas de especialidad y servicios diversos (López Lucio, 2006). Esta realidad se pone de manifiesto en las parcelas de uso comercial exclusivo. Aunque poco representativas en número, se caracterizan por su mayor superficie relativa de suelo ocupado y edificado. En menor medida, este suelo comercial periférico no residencial se asocia a las oficinas y la industria.

6. La función industrial destaca por su carácter exclusivo y su débil capacidad de mezcla con otros usos, como corresponde a los polígonos industriales 0 espacios especializados del territorio donde se concentra un cierto número de empresas dedicadas a este tipo de actividad. Así, en el $88,4 \%$ del total de las parcelas edificadas, dedicadas al sector de la industria, predomina este uso exclusivo, como corresponde a la tradición manufacturera de este municipio de la periferia madrileña.

7. Finalmente, podemos destacar la tendencia del uso del suelo destinado a oficinas a mezclarse con otros usos. En el conjunto de ambos sectores espaciales, únicamente el $19,2 \%$ de las parcelas es de uso exclusivo, destacando la asociación con el resto de los usos. 


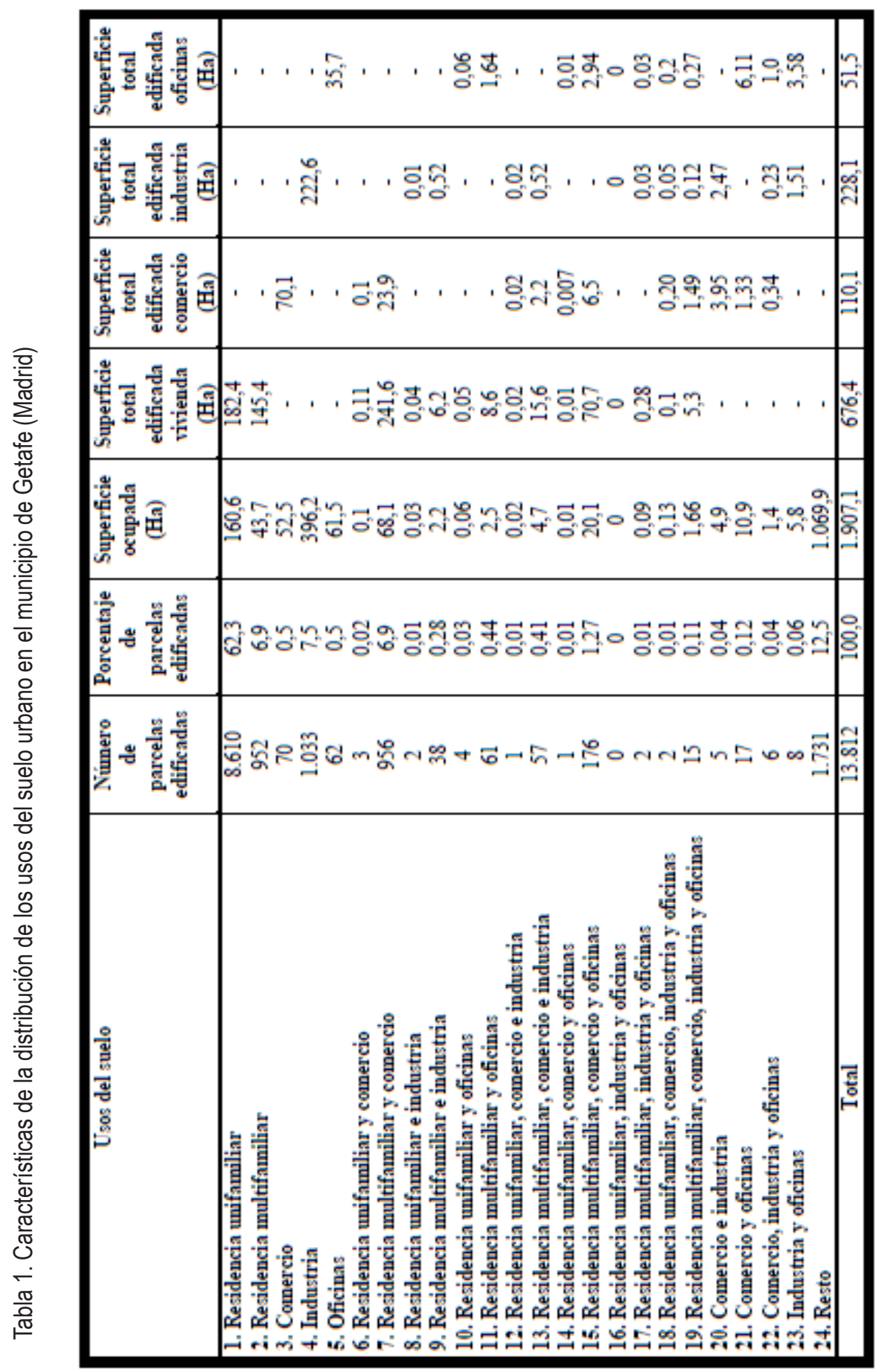




\section{DEFINICIÓN DEL PATRÓN DE OCUPACIÓN TERRITORIAL DEL MUNICIPIO DE GETAFE (MADRID), DE ACUERDO AL MODELO DE LA CIUDAD DISPERSA}

La propuesta de usos del suelo base, con vistas a la obtención de patrones reales de ocupación del territorio, la vamos a realizar, a partir de los dos elementos utilizados en el apartado anterior: unidad territorial de análisis y categoría de uso del suelo. La unidad espacial ha sido, en nuestro caso, la parcela catastral, mientras que para las categorías de ocupación del suelo hemos seleccionado, exclusivamente, los usos más representativos. De acuerdo a este planteamiento simplificador, proponemos la siguiente categorización (Tabla 2), por agrupación de clases, en la perspectiva de obtener los denominados "espacios de vocación" (Bozzano et al., 2008).

Tabla 2. Nuevas clases del uso del suelo urbano por agrupamiento de las categorías más simples

\begin{tabular}{|c|c|}
\hline Nuevas categorías & Categorías agrupadas \\
\hline 1. Residencial unifamiliar exclusivo & 1 \\
\hline 2. Residencial unifamiliar dominante & $6,8,10,12,14,16$ y 18 \\
\hline 3. Residencial multifamiliar exclusivo & 2 \\
\hline 4. Residencial multifamiliar dominante & $7,9,11,13,15,17$ y 19 \\
\hline 5. Comercial exclusivo & 3 \\
\hline 6. Industrial exclusivo & 4 \\
\hline 7. Uso de oficinas exclusivo & 5 \\
\hline 8. Uso mixto (comercio, industria y oficinas) & $20,21,22$ y 23 \\
\hline
\end{tabular}

Los espacios vocacionales pueden ser definidos como lugares en términos de patrones de ocupación y apropiación territorial, donde se identifican matices y variantes en cada vocación. Aunque no corresponden a un único uso del suelo, predomina en ellos una actividad sobre las demás. Así, por vocación residencial multifamiliar dominante se entendería el lugar donde predominaran usos y funciones destinadas a la vivienda de uso permanente o temporal de varias familias, sobre otras vocaciones, como el comercio, la industria artesanal o cualquier otra actividad que pudiera considerarse complementaria.

La reconstrucción de las unidades territoriales formales o piezas del mosaico urbano, a partir de las unidades elementales funcionales o parcelas, requiere del conocimiento preciso de los procesos acaecidos en la ciudad, ya que son éstos los que permiten interpretar los espacios conformados como una consecuencia de la dinámica del desarrollo urbano, en concordancia con las necesidades del sistema productivo, los gustos demandados por los consumidores y los avances de las 
nuevas tecnologías.

El desarrollo urbano de las grandes ciudades de nuestro país y la configuración de su estructura más reciente no ha tenido lugar de forma brusca, sino progresiva, desde los primeros momentos de conformación del modelo metropolitano clásico hasta el modelo disperso actual. Font et al. (1999) diferencian, al respecto, varios patrones de cambio clave en la presente disposición de "la estructura polinuclear discontinua" de nuestras metrópolis, que han dejado su impronta sobre el territorio:

a) La expansión del núcleo central y de los núcleos históricos de la región metropolitana, con caracteres de continuidad espacial y promiscuidad de usos (residencial, industrial, servicios, etc.), resultado de procesos de extensión y densificación de las mallas existentes, que fueron característicos hasta principios de los años setenta del pasado siglo. Estos ensanches de los núcleos urbanos de la primera y segunda coronas metropolitanas aún están presentes en los principales municipios de las aglomeraciones urbanas españolas.

b) Los nuevos emplazamientos o lugares de las actividades de la innovación o de la centralidad dispersa, en enclaves de alta accesibilidad o exposición visual, o a lo largo de los elementos principales de la infraestructura viaria y del transporte público. Las grandes superficies comerciales, los grandes equipamientos y dotaciones (hospitales, universidades, instalaciones deportivas, etc.) formarían parte de estas nuevas geografías urbanas.

c) Finalmente, las formas del crecimiento disperso, tradicionalmente la edificación aislada de la segunda residencia, que además de seguir consumiendo importantes superficies del territorio metropolitano, se han convertido, en parte, en residencia permanente en los territorios próximos.

Sirviéndonos de esta referencia teórica, nos proponemos la comprobación, en el territorio del municipio de Getafe, de la existencia de alguna de estas unidades básicas, que, a modo de ensayo, nos ofrezcan la línea a seguir en el intento de reconstruir el entramado global de la estructura de toda la urbe.

\subsection{Delimitación de algunos patrones de ocupación territorial, en el municipio de Getafe (Madrid)}

Como ya hemos indicado, el conocimiento de los procesos de transformación urbana ayuda sobremanera a identificar las unidades morfo funcionales de 
la estructura de la ciudad, que deben ser analizadas, de manera evolutiva en el tiempo, desde la expansión u ocupación cada vez más periférica del espacio. En esta perspectiva evolutiva del análisis de la estructura de la ciudad actual, debemos concentrarnos en dos momentos clave que diferencian unidades territoriales específicas. En general, las transformaciones acaecidas durante los años sesenta y setenta del pasado siglo, en el momento de conformación del área metropolitana madrileña, tuvieron como consecuencia la creación de zonas funcionalmente mixtas, en los cascos antiguos y áreas de expansión de los municipios cercanos a la ciudad central, y de áreas homogéneas en la periferia de los mismos (residenciales de alta densidad e industriales). Por otra parte, los procesos de dispersión de la ciudad, connaturales con el crecimiento urbano del momento presente, se identifican más con la existencia de áreas homogéneas residenciales de baja densidad (tanto unifamiliares como multifamiliares), nodos o enclaves del terciario o de la industria de innovación (parques tecnológicos), asentamientos industriales dispersos, grandes equipamientos y dotaciones (hospitales, universidades, parques regionales), etc.

En este intento de identificar y delimitar patrones de ocupación del suelo en el municipio de Getafe (Madrid), a modo de entramados urbanos homogéneos, siguiendo la terminología explícita de la obra antes reseñada, hemos seleccionado tres espacios geográficos perfectamente diferenciados, que muestran la profunda transformación funcional de la ciudad actual acorde con el nuevo modelo de ciudad: el barrio de Juan de la Cierva, cuya configuración urbana tuvo lugar durante los años setenta del pasado siglo; el polígono industrial de Los Ángeles, surgido en el mismo periodo de tiempo, y la urbanización Sector 3 , de origen más reciente, una de las urbanizaciones residenciales de baja densidad más extensa de dicho municipio (Figura 2). 
Figura 2. Situación geográfica de los tres espacios geográficos seleccionados para el análisis en el municipio de Getafe (Madrid).

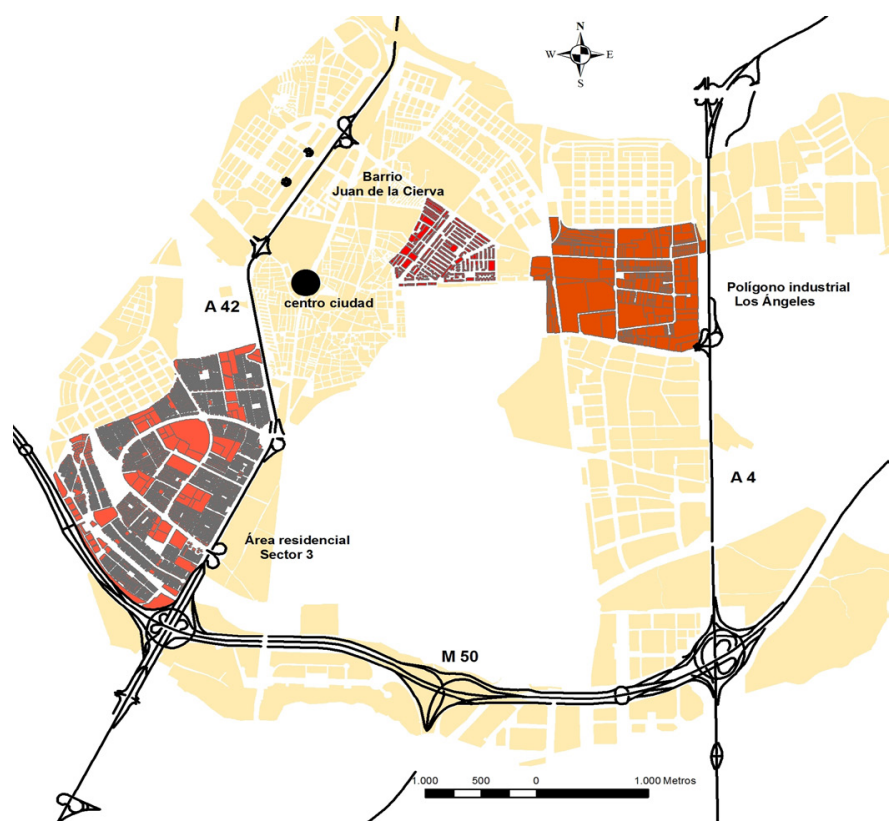

El barrio de Juan de la Cierva: zona residencial de la primera corona metropolitana madrileña

El barrio de Juan de la Cierva constituye parte esencial de la expansión del municipio de Getafe durante los años setenta del pasado siglo (Figura 3). Su desarrollo urbano ha estado ligado, por tanto, al momento del crecimiento de los municipios de la primera corona metropolitana madrileña, como consecuencia de la profunda crisis de la agricultura tradicional, motivada por la introducción de nuevas técnicas y procesos productivos, que convirtieron las áreas rurales en espacios de paro estructural. En estas condiciones, el fuerte contingente inmigratorio, que atraído por la oferta laboral de un mercado de trabajo expansivo llegaba a la ciudad de Madrid, tuvo que ser realojado en un tiempo récord, desencadenando procesos intensivos de ocupación del espacio que originaron cambios cualitativos en las formas y modos de producción urbana. El nuevo ensanche urbano fue construido en bloques de pisos no demasiado elevados (por la proximidad del aeródromo), generalmente de cuatro alturas, alcanzando densidades de ocupación del suelo muy altas, que superaban, en ocasiones, las 250 viviendas por hectárea.

Los resultados obtenidos de la medición de la estructura funcional de esta 
área ${ }^{4}$ vienen reflejados en la Tabla 3 y corroboran que el barrio de Juan de la Cierva presente las características propias de los primitivos ensanches residenciales que compactaron el espacio próximo a los cascos antiguos de los municipios de la primera corona metropolitana del Sur de Madrid. En su estructura urbana, destaca el predominio funcional residencial, típico de las ciudades dormitorio de la periferia metropolitana de Madrid, con una fuerte relación con el comercio, ya que en más del $60 \%$ de las parcelas urbanas se integran los edificios multi vivienda con los establecimientos comerciales (Figura 3). Se codifica así el modelo de tejido residencial multifuncional, con predominio de la vivienda como elemento más representativo, soporte de la estructura comercial tradicional basada en la calle. Asimismo, aunque en un segundo plano, apreciamos otras formas de integración de la función residencial con otras actividades a ella asociadas, como el pequeño terciario de oficinas o servicios personales, los talleres de reparación o los pequeños equipamientos, aunque, como puede observarse por la superficie edificada, escasamente representadas.

Figura 3. Distribución espacial de los usos del suelo del barrio de Juan de la Cierva

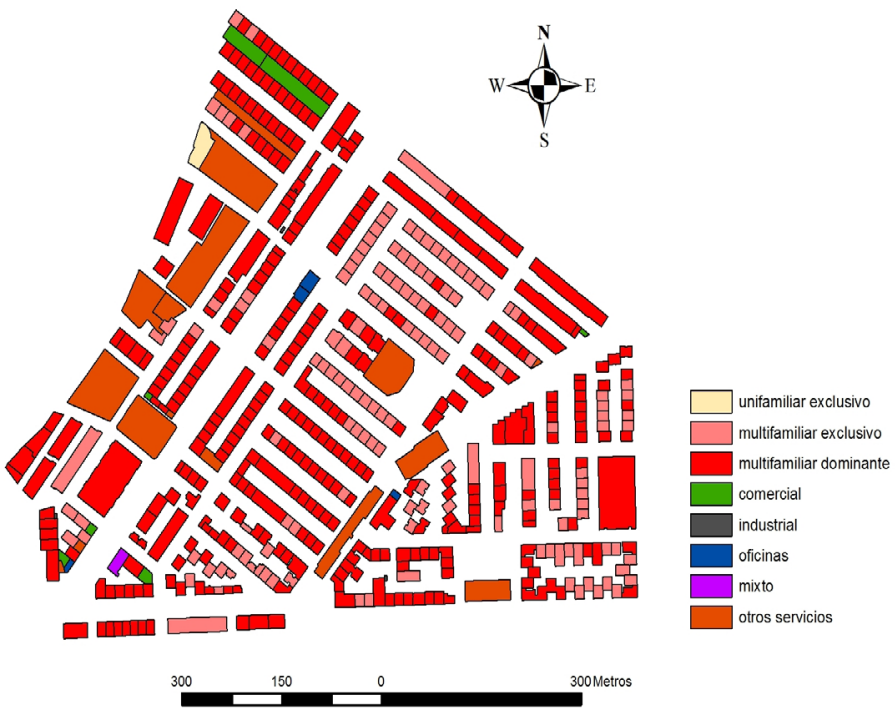

\footnotetext{
${ }^{4}$ Con esta intención, se ha seleccionado, a partir de la base de datos municipal, la correspondiente al barrio de Juan de la Cierva, exportando la base de datos temática dBase del SIG al programa Statgraphics Centurión, donde se ha realizado un tratamiento estadístico de la información. Esta operación ha consistido en reagrupar las categorías iniciales de los usos del suelo, midiendo el número de parcelas, superficie de suelo ocupada, superficie edificada por las nuevas categorías y promedio del número de viviendas y establecimientos productivos por parcela.
} 
Tabla 3. Características funcionales del barrio de Juan de la Cierva (Getafe)

\begin{tabular}{||c|c|c|c|c|c|c||}
\hline \multirow{2}{*}{ Uso suelo } & \multicolumn{2}{|c|}{ Parcelas } & \multicolumn{4}{c||}{ Uso suelo edificado (ha) } \\
\cline { 2 - 7 } & número & porcentaje & residencial & comercial & industrial & oficinas \\
\hline $\mathbf{1}$ & 1 & 0,2 & 0,12 & - & - & - \\
$\mathbf{3}$ & 148 & 28,1 & 22,2 & - & - & - \\
$\mathbf{4}$ & 346 & 65,8 & 62,6 & 7,0 & - & 0,75 \\
$\mathbf{5}$ & 7 & 1,3 & - & 1,0 & - & - \\
$\mathbf{6}$ & 3 & 0,6 & - & - & 0,26 & - \\
$\mathbf{7}$ & 4 & 0,8 & - & - & - & 0,06 \\
$\mathbf{8}$ & 1 & 0,2 & - & 0,9 & 0,008 & 0,14 \\
$\mathbf{9}$ & 16 & 3,0 & - & - & - & - \\
\hline
\end{tabular}

1. Residencial unifamiliar exclusivo 2. Residencial unifamiliar dominante 3. Residencial multifamiliar exclusivo 4. Residencial multifamiliar dominante 5. Comercial exclusivo 6. Industrial exclusivo 7. Uso de oficinas exclusivo 8 . Usos mixtos (comercio, industria y oficinas) 9. Otros servicios.

\section{El polígono industrial de Los Ángeles}

El polígono de Los Ángeles es uno de los polígonos industriales más antiguos, grandes e importantes de la ciudad. Se encuentra al este del centro urbano, al borde de la autovía A4 (Madrid-Andalucía), a $13 \mathrm{~km}$ del centro de Madrid. El surgimiento de este polígono industrial tuvo lugar gracias a la aprobación del plan parcial de Los Ángeles, como desarrollo del Plan General de Ordenación Urbana de 1968, debido a la necesidad de suelo industrial en la periferia madrileña. La nueva orientación hacia un modelo más descentralizado de la industria se apoyaba en varios factores. En primer lugar, el precio del suelo, mucho más reducido en esta zona periférica de la ciudad de Madrid. En segundo lugar, las nuevas exigencias tecnológicas de la fabricación resultaron fundamentales, ante la necesidad de un mayor tamaño por planta y una mayor potencia instalada, no disponible en el centro de la capital. Finalmente, fue decisivo el desarrollo experimentado por el sector del transporte, sobre todo del transporte por carretera, que facilitaba una relación más fluida y versátil con otras zonas urbanas metropolitanas, merced a la existencia de carreteras radiales y de circunvalación.

Desde sus inicios, el polígono industrial de Los Ángeles (Figura 4) fue construido con parcelas pensadas para alojar grandes fábricas y almacenes, principalmente del sector metalmecánico, sin apenas otras preocupaciones urbanísticas y ambientales que la proximidad a la autovía A4 y unos precios del suelo moderados. Para ello, contaba con algunas de las mayores empresas del municipio (Uralita, Arcelor, Radisa, 
etc.). Esta situación ha cambiado en la actualidad debido a un proceso de tercerización económica y de progresiva especialización industrial a favor de sectores más intensivos en saber, una mayor cualificación laboral y una diversificación de la estructura social del municipio. Los datos de la tabla 4, deducidos de la información catastral, muestran actualmente al polígono como una zona fundamentalmente industrial, cuyo suelo urbano destinado al sector fabril ocupa 67,8 ha, la mayor parte de la superficie existente. De la misma, se ha segregado, prácticamente en su totalidad, la función residencial, que apenas se encuentra representada. No así otras funciones como el comercio y sobre todo las oficinas, cuyo crecimiento se ha visto acompañado por una paralela renovación de la oferta inmobiliaria para las empresas, que intenta ahora atraer actividades de mayor rango y escaso impacto ambiental en espacios de cierta calidad urbanística. De esta manera, se explica que se ubique, aquí, el Centro Municipal de Empresas, que actúa como vivero empresarial, y un Centro de Teletrabajo, que ha albergado hasta el momento a 81 empresas, generadoras de más de 400 empleos directos.

Tabla 4. Características funcionales del polígono industrial de Los Ángeles

\begin{tabular}{||c|c|c|c|c|c|c||}
\hline \multirow{2}{*}{ Uso suelo } & \multicolumn{2}{|c|}{ Parcelas } & \multicolumn{4}{c||}{ Uso suelo edificado (ha) } \\
\cline { 2 - 7 } & número & porcentaje & residencial & comercial & industrial & oficinas \\
\hline $\mathbf{3}$ & 1 & 0,3 & 0,04 & - & - & - \\
$\mathbf{5}$ & 1 & 0,3 & - & 0,14 & - & - \\
$\mathbf{6}$ & 255 & 76,8 & - & - & 67,8 & - \\
$\mathbf{7}$ & 10 & 3,0 & - & - & - & 2,0 \\
$\mathbf{8}$ & 3 & 0,9 & - & 0,05 & 0,8 & 1,0 \\
$\mathbf{9}$ & 62 & 18,7 & - & - & - & - \\
\hline
\end{tabular}

1. Residencial unifamiliar exclusivo 2. Residencial unifamiliar dominante 3. Residencial multifamiliar exclusivo 4. Residencial multifamiliar dominante 5. Comercial exclusivo 6. Industrial exclusivo 7. Uso de oficinas exclusivo 8. Usos mixtos (comercio, industria y oficinas) 9. Otros servicios. 
Figura 4. Distribución espacial de los usos del suelo del polígono industrial de Los Ángeles en el municipio de Getafe (Madrid).

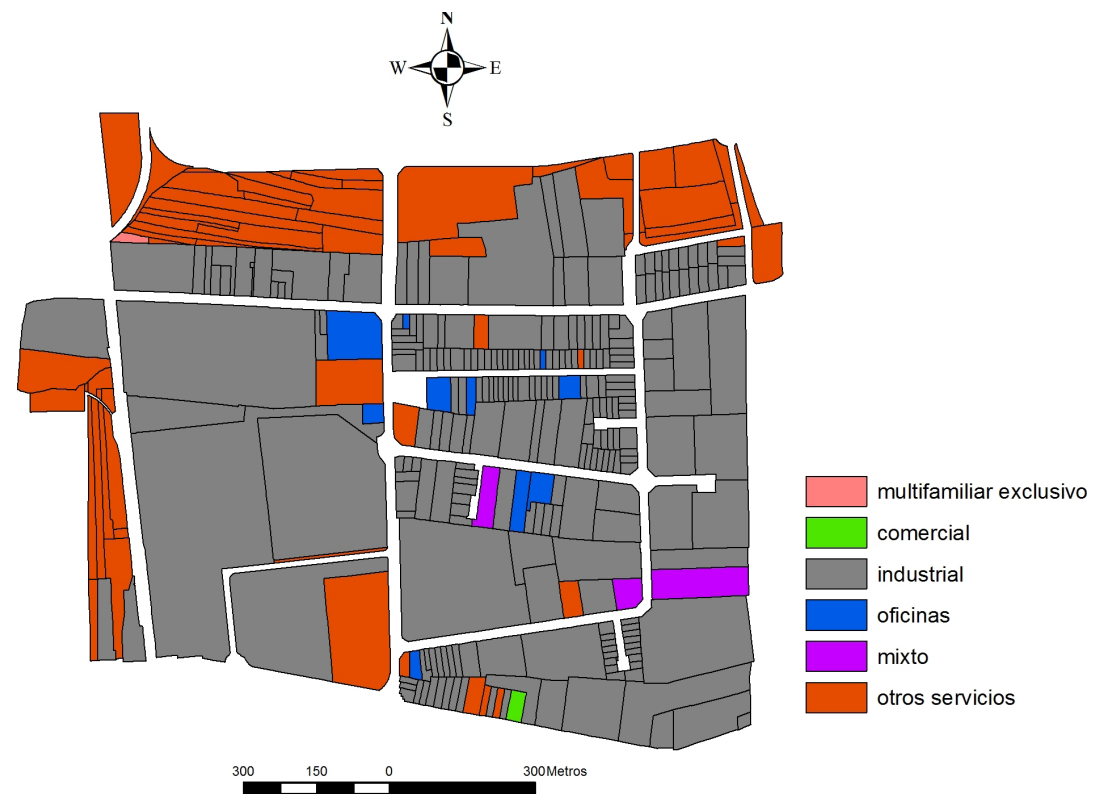

La urbanización residencial extensiva del Sector 3

La urbanización residencial del Sector 3 se inició como un proyecto singular por su concepción, puesto que rompía la tradicional vinculación entre tipología edificatoria y condición social. Sus promotores fueron grupos sindicalistas, decididos a poner al servicio de la clase obrera, mediante régimen de cooperativa, viviendas unifamiliares con jardín, en una forma de vida ligada, hasta ese momento, a clases de mayor nivel de renta. La ejecución del proyecto del Sector 3, desarrollado a lo largo de los años ochenta y noventa del pasado siglo, enlaza con el modelo de ciudad dispersa, ya que la idea igualitaria primitiva cedió paso con el tiempo a la especulación urbanística y a la ocupación del espacio por clases medias más acomodadas.

El espacio ocupado por el barrio está separado del resto urbanizado del municipio por la autovía A 42 (carretera de Madrid-Toledo). Las características funcionales de la urbanización (Tabla 5) muestran el predominio, casi exclusivo, de la función residencial de baja densidad, con viviendas unifamiliares, cuya participación supera el 97\% del número de parcelas existentes. Así mismo, la vivienda unifamiliar consume más del 
$70 \%$ de la superficie edificada, respecto a los otros usos del suelo allí representados, debido a las mayores necesidades de suelo que este tipo de vivienda representa en relación con la plurifamiliar o en bloque. Esta realidad manifiesta una de las propiedades del espacio residencial extensivo, en el que progresivamente se ha ido clausurando la mezcla con otros usos. El tejido residencial multifamiliar también aparece, aunque en mucha menor proporción. La zona más representativa del mismo ocupa el denominado Arroyo Culebro, situado en el extremo sur del Sector 3, limitando con la autovía M-50, en una zona mixta, integrada por urbanizaciones de chalets adosados y bloques de pisos.

El área residencial se complementa con zonas comerciales y de otros servicios (sobre todo sanitarios, educativos), como corresponde a un barrio bien equipado de nivel social medio alto. Las áreas comerciales son, en su mayor parte, de uso exclusivo, como el Centro Comercial Getafe 3, que dispone de aproximadamente 8 ha comerciales, destinadas a hipermercado, y tiendas de moda y complementos del hogar, bricolaje y electrodomésticos, establecimientos de restauración y ocio, y actividades diversas, con escasa diversificación de usos de suelo, acorde con el nuevo modelo disperso de ciudad.

Tabla 5. Características funcionales de la zona residencial

Sector 3 de Getafe (Madrid)

\begin{tabular}{||c|c|c|c|c|c|c||}
\hline \hline \multirow{2}{*}{ Uso suelo } & \multicolumn{2}{|c|}{ Parcelas } & \multicolumn{4}{c||}{ Uso suelo edificado (ha) } \\
\cline { 2 - 7 } & número & porcentaje & residencial & comercial & industrial & oficinas \\
\hline $\mathbf{1}$ & 6.411 & 97,3 & 123,8 & - & - & - \\
$\mathbf{2}$ & 16 & 0,24 & 8,90 & - & - & - \\
$\mathbf{3}$ & 4 & 0,06 & 3,78 & - & - & - \\
$\mathbf{4}$ & 15 & 0,23 & - & 0,17 & - & - \\
$\mathbf{5}$ & 46 & 0,70 & - & 16,2 & - & - \\
$\mathbf{6}$ & 4 & 0,06 & - & - & 0,30 & - \\
$\mathbf{7}$ & 5 & 0,08 & - & - & - & 0,66 \\
$\mathbf{8}$ & 39 & 0,60 & - & 0,60 & 0,07 & 0,13 \\
$\mathbf{9}$ & 51 & 0,77 & - & - & - & - \\
\hline
\end{tabular}

1. Residencial unifamiliar exclusivo 2. Residencial unifamiliar dominante 3. Residencial multifamiliar exclusivo 4. Residencial multifamiliar dominante 5. Comercial exclusivo 6. Industrial exclusivo 7. Uso de oficinas exclusivo 8. Usos mixtos (comercio, industria y oficinas) 9. Otros servicios. 
Figura 5. Distribución espacial de los usos del suelo de la zona residencial Sector 3 de Getafe (Madrid)

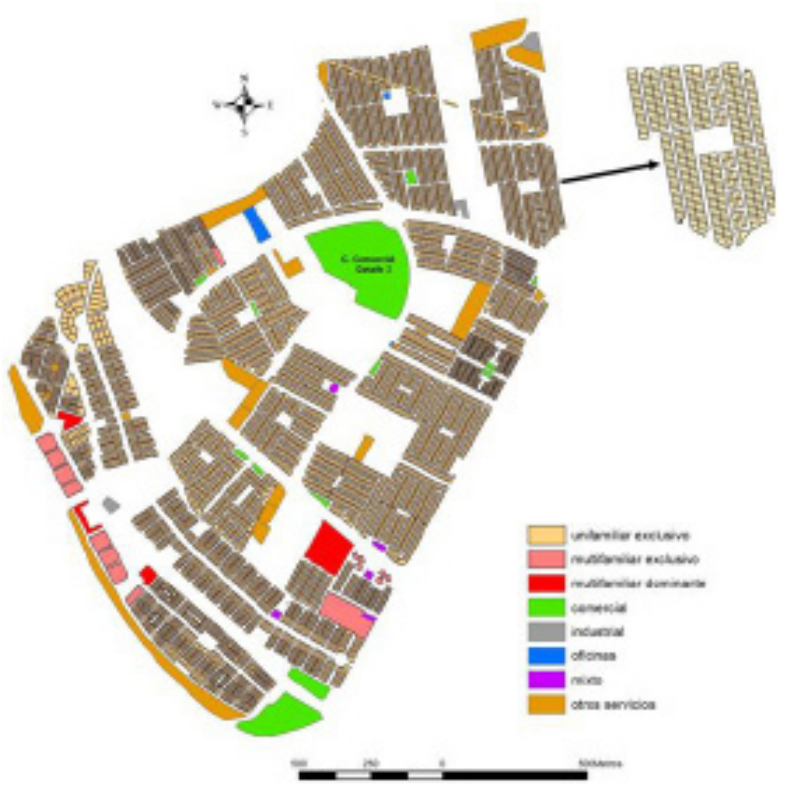

\section{CONCLUSIONES}

La utilización del catastro de urbana en el estudio del territorio ofrece nuevas perspectivas de gran interés, en fines tan diversos como la valoración de su problemática, la cartografía o representación del mismo, y, finalmente, la ordenación y planificación territorial. Su trascendencia como tal se deriva de la capacidad de la parcela urbana, para integrar, en un espacio físico reducido, un conjunto de elementos clave para interpretar la ciudad, desde la perspectiva de identificar la lógica de los fenómenos del crecimiento urbano. En nuestro caso, la metodología desarrollada a partir de usos del suelo urbano, incluidos con carácter multifuncional, muestra la posibilidad de analizar, cuantitativamente, la estructura de nuestras urbes, de manera evolutiva en el tiempo, permitiendo realzar los profundos contrastes existentes entre la ciudad compacta del pasado y la ciudad dispersa actual, a partir del mosaico urbano y las piezas funcionales asociadas a cada una de los espacios de actividad, consumo y ocio que lo integran. 


\section{BIBLIOGRAFÍA}

- Bozzano, H., Carut, C., Barbetti, G. C. y Arrivillaga, N. (2008). "Usos del suelo y lugares: criterios teórico-metodológicos. Aplicación a un caso en Guatemala", Revista Universitaria de Geografía (on line), nº 17, pp. 189-231.

- De Santiago Rodríguez, E. (2008). "Nuevas formas y procesos espaciales en el territorio contemporáneo: la 'ciudad única"', Polis. Vol. 7, $n^{0} 20$. Editorial de la Universidad Bolivariana, Chile, pp. 53-71.

- Dematteis G. (1998). "Suburbanización y periurbanización. Ciudades anglosajonas y ciudades latinas", La ciudad dispersa: suburbanización y nuevas periferias, F. J. Monclús (Ed). Centro de Cultura Contemporánea. Barcelona, pp. $5-15$.

- Feria Toribio, J. M. (1999). "Nuevas periferias urbanas y planificación pública", La ciudad: tamaño y crecimiento. III Coloquio de Geografía Urbana. Antequera (Málaga), pp. 309-316.

- Ferrás Sexto, C. (2000). "Ciudad dispersa, aldea virtual y revolución tecnológica. Reflexión acerca de sus relaciones y significado social", Scripta Nova, Revista Electrónica de Geografía y Ciencias Sociales. Disponible en: http://www.ub.es/geocrit/ sn-69-68.htm. Universidad de Barcelona.

- Font, A. (1997). "Anatomía de una metrópoli discontinua: la Barcelona metropolitana”, Papers, Región Metropolitana de Barcelona, n² 26, pp. 9-19, Barcelona.

- Font, A., Llop, C. y Vilanova, J. M. (1999). La construcción del territorio metropolitano. Morfogénesis de la región urbana de Barcelona. Barcelona: Mancomunitat Metropolitana de Municipis.

- López Lucio, L. (2006). “Espacio público e implantación comercial en la ciudad de Madrid", Cuadernos de investigación urbanística, $n^{0}$ 23, Madrid, pp. 1-48.

- Nel.lo, O. (1998). "Los confines de la ciudad sin confines. Estructura urbana y límites administrativos en la ciudad difusa", en Monclus. J. (Ed) La ciudad dispersa. Barcelona: Centro de Cultura Contemporània de Barcelona.

- $\quad$ Rhind, D. y Hudson, R (1980). Land Use. Methuen, Nueva York. 